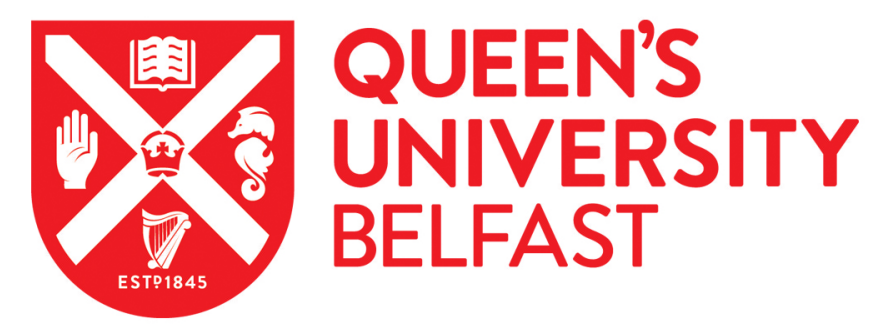

\title{
Surface-enhanced Raman Spectroscopy for the Detection of a Metabolic Product in the Headspace Above Live Bacterial Cultures
}

\author{
Kelly, J., Patrick, R., Patrick, S., \& Bell, S. E. J. (2018). Surface-enhanced Raman Spectroscopy for the \\ Detection of a Metabolic Product in the Headspace Above Live Bacterial Cultures. Angewandte Chemie \\ International Edition, 57(48), 15686. https://doi.org/10.1002/anie.201808185
}

Published in:

Angewandte Chemie International Edition

Document Version:

Peer reviewed version

Queen's University Belfast - Research Portal:

Link to publication record in Queen's University Belfast Research Portal

Publisher rights

(c) 2018 WILEY-VCH Verlag GmbH \& Co. KGaA, Weinheim. This work is made available online in accordance with the publisher's policies. Please refer to any applicable terms of use of the publisher.

\section{General rights}

Copyright for the publications made accessible via the Queen's University Belfast Research Portal is retained by the author(s) and / or other copyright owners and it is a condition of accessing these publications that users recognise and abide by the legal requirements associated with these rights.

Take down policy

The Research Portal is Queen's institutional repository that provides access to Queen's research output. Every effort has been made to ensure that content in the Research Portal does not infringe any person's rights, or applicable UK laws. If you discover content in the Research Portal that you believe breaches copyright or violates any law, please contact openaccess@qub.ac.uk. 


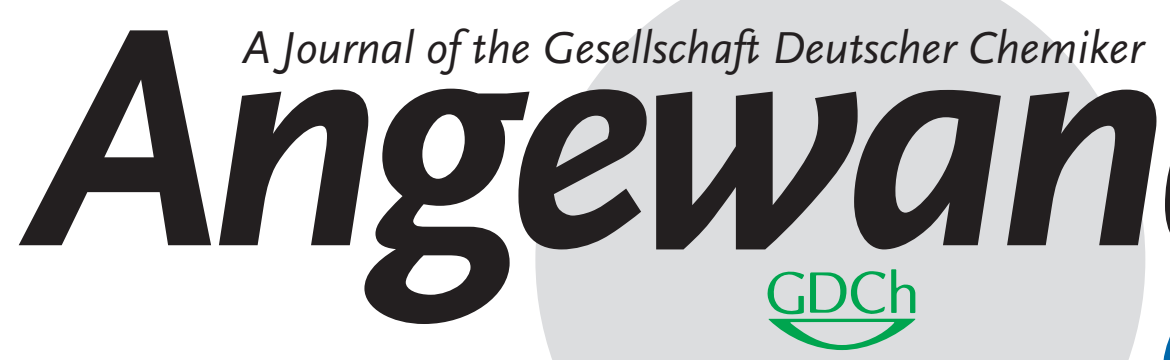

International Edition

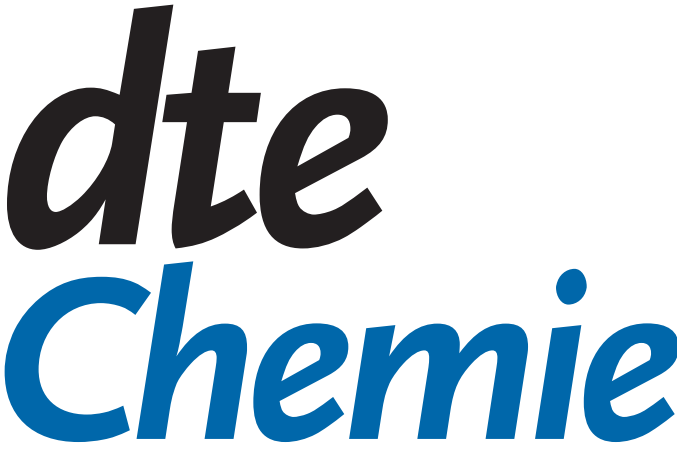

www.angewandte.org

\section{Accepted Article}

Title: Surface-enhanced Raman Spectroscopy for the Detection of a Metabolic Product in the Headspace Above Live Bacterial Cultures

Authors: Jessica Kelly, Robin Patrick, Sheila Patrick, and Steven E. J. Bell

This manuscript has been accepted after peer review and appears as an Accepted Article online prior to editing, proofing, and formal publication of the final Version of Record (VoR). This work is currently citable by using the Digital Object Identifier (DOI) given below. The VoR will be published online in Early View as soon as possible and may be different to this Accepted Article as a result of editing. Readers should obtain the VoR from the journal website shown below when it is published to ensure accuracy of information. The authors are responsible for the content of this Accepted Article.

To be cited as: Angew. Chem. Int. Ed. 10.1002/anie.201808185

Angew. Chem. 10.1002/ange.201808185

Link to VoR: http://dx.doi.org/10.1002/anie.201808185

http://dx.doi.org/10.1002/ange.201808185 


\title{
Surface-enhanced Raman Spectroscopy for the Detection of a Metabolic Product in the Headspace Above Live Bacterial
} Cultures

\author{
Jessica Kelly, ${ }^{[a]}$ Dr. Robin Patrick, ${ }^{[a]}$ Prof. Sheila Patrick, ${ }^{[b]}$ Prof. Steven E.J. Bell ${ }^{[a] *}$
}

\begin{abstract}
In-situ surface-enhanced Raman spectroscopy of the headspace above cultures of 6 bacterial species allowed sensitive and selective detection of characteristic bands from chemisorbed methyl sulphide. This marker compound is created by dissociation of adsorbed dimethyl disulphide (DMDS) on the surface of the Au or Ag nanoparticle (NP) films which are used as the enhancing media. DMDS is a fermentative metabolite of bacteria and was detected here by GC-MS of the headspace of bacteria cultures. Kinetic binding plots of media spiked with DMDS and of live cultures, both showed that the more rapid adsorption of DMDS onto Au based substrates made them more suitable for the rapid detection of bacteria than $\mathrm{Ag}$ based substrates. E. coli DH5a was chosen for the proof-of-principal experiments. For this micro-organism, the sensitivity limit was found to be the detection of bacteria in the headspace of a $1.5 \times 10^{7} \mathrm{CFU} / \mathrm{ml}$ culture. Under our conditions, this corresponds to detection 15 minutes after inoculation of the growth medium, but it will depend on the CFU/ml of the initial inoculum used. Since the metabolites are only produced by viable bacteria, antibiotic (gentamicin) treatment stopped the normal signal growth of the marker peak. This work is a promising step towards rapid bedside detection of bacterial infections and rapid screening of antibiotics against model cultures.
\end{abstract}

Rapid, low-cost testing for viable bacteria has several potential applications, the most obvious would be as a simple diagnostic tool but it also has considerable potential for testing antibiotic susceptibility, since it would provide an alternative to the culture based methods which are now widely used within clinical microbiology laboratories to directly support the care of patients with bacterial infections. More broadly, faster, cheaper methods to monitor the effectiveness of antibiotics can help to combat antimicrobial resistance (AMR) through reducing over-use of antibiotics, particularly in resource-poor healthcare environments. ${ }^{[1]}$ The gold standard method for testing the viability of bacteria is through culture based techniques but this approach can have a long turnaround time, ranging from days to weeks. ${ }^{[2]}$ Real time PCR is widely used to detect and identify bacteria and can give results in a few hours but the standard approach does not discriminate between viable and non-viable bacteria. ${ }^{[3]}$ Live/dead staining using dual dyes is also used but again requires sophisticated laboratory based instrumentation (flow cytometers or fluorescence imaging microscopes) ${ }^{[4]}$ and specialist operators. Here, we show that surface-enhanced

[a] J. Kelly, Dr. R. Patrick, Prof. S. E. J. Bell

School of Chemistry \& Chemical Engineering

Queen's University Belfast BT9 5AG (UK)

E-mail: s.bell@qub.ac.uk

[b] Prof. S. Patrick

School of Medicine, Dentistry and Biomedical Sciences

Centre for Infection and Immunity

Queen's University Belfast BT9 5AG (UK)

Supporting information for this article is given via a link at the end of the document.
Raman (SERS) spectra of the headspaces above bacterial cultures show strong characteristic vibrational bands which are due to the chemisorption of dimethyl disulphide (DMDS). Since DMDS is a bacterial metabolite and therefore indicative of metabolically active cultures, this provides a simple and rapid method for detecting viable bacterial cultures. Other vibrational spectroscopic techniques, such as Raman micro-spectroscopy, along with isotope probing have been widely used for detecting microbial metabolic activity and its response to antibiotics. ${ }^{[5],[6],[7]}$ In that work, however, the emphasis is on understanding rather than simple detection since the measurements typically begin with known micro-organisms.

Previously, gas chromatography-mass spectrometry (GC$\mathrm{MS}),{ }^{[8]}$ ion mobility spectrometry $(\mathrm{IMS})^{[9]}$ or proton transfer reaction-mass spectrometry (PTR)-MS ${ }^{[10]}$ have been used to monitor bacterial headspace and there are numerous publications ${ }^{[11],[12],[13]}$ available on the detection and identification of the metabolites produced from bacteria using GC-MS in particular. However, these approaches are not suitable for bedside measurements and have not been used to determine cell viability. Alternatively, portable chemical sensors such as electronic noses (e-nose) have been reported to detect bacterial volatiles with high sensitivity. ${ }^{[14],[15],[16]}$ However, the detection and identification of bacterial metabolites using these sensors is much more complex than that used in this work, since e-nose systems are based on pattern recognition and are therefore poor at detecting analytes of an unknown identity. Unlike GC-MS, enoses are not an universally applicable gas sensor, since the proper selection of an appropriate e-nose for a certain application must include a detailed evaluation of operating conditions of the sensor in the array and the composition of analytes being detected. Conversely, SERS has the sensitivity that previous studies suggest is needed to detect headspace volatiles but also combines this with the ability to make rapid, non-invasive measurements, which can act as a general marker for live bacteria, using relatively low-cost hand-held instruments. ${ }^{[17]}$

The six micro-organisms used in the study: Escherichia coli DH5a, Escherichia coli K12 WT, Staphylococcus aureus Cowan I, Enterococcus faecalis ATCC 10541, Pseudomonas aeruginosa OA1 and Bacteroides fragilis NCTC 9343 were obtained from the micro-organism collection of the Centre for Infection and Immunity, Queens University Belfast.

In-situ SERS monitoring of the headspace above the cultures used a SERS substrate composed of a densely-packed monolayer of dry $\mathrm{Ag}$ or $\mathrm{Au}$ particles deposited onto a quartz window from a metal-like liquid film created by assembling the particles at a water/dichloromethane interface. ${ }^{[18]}$ Data on characterisation of the $\mathrm{Ag}$ and $\mathrm{Au}$ substrates can be found in the Supporting Information (Figure S1). Since the films were optically transparent, they could be fixed onto the cap of the growth vial, as shown in Figure 1, with the particle layer facing 
the growth medium, so that the SERS signals could be monitored from the reverse side while keeping the vial capped. SERS spectra were recorded using a hand-held Raman spectrometer which was mounted above the vial within an aerobic or anaerobic incubator at $37{ }^{\circ} \mathrm{C}$, allowing continuous monitoring of the headspace right from initial inoculation of the culture medium.

Since numerous volatile compounds have been reported in the headspace of bacteria, ${ }^{[13]}$ it was expected that the SERS spectra might show numerous peaks associated with a complex mixture of analytes. In fact, as shown in Figure 1, the SERS spectra recorded with the $\mathrm{Ag}$ substrate in the $400-2200 \mathrm{~cm}^{-1}$ range, showed only one new band at $674 \mathrm{~cm}^{-1}$, in addition to the citrate signal from the blank substrate (control SERS experiments of the headspace of the sterile culture media using the $\mathrm{Ag}$ substrate are shown in the Supporting Information, Figure S2). The spectra in Figure 1 also show that this additional band was detected when the bacteria were cultured in different types of media.

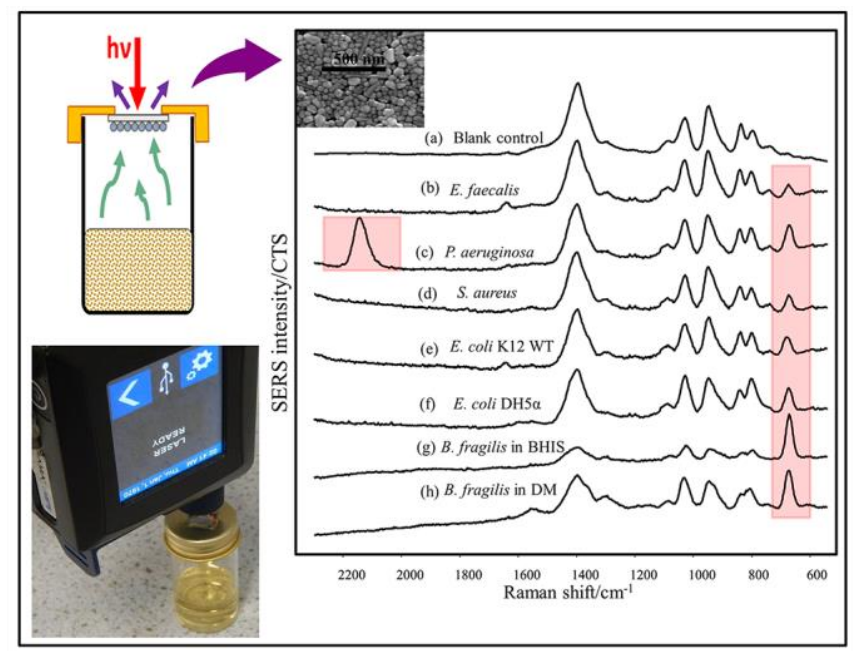

Figure 1: SERS spectra of the headspace of (a) blank control (b) E. faecalis ATCC 10541 (c) P. aeruginosa OA1 (d) S. aureus Cowan I (e) E. coli K12 WT (f) E. coli $\mathrm{DH} 5 \mathrm{a}(\mathrm{g})$ B. fragilis NCTC 9343 cultivated in Brain Heart Infusion Broth supplemented (BHIS) (h) B. fragilis NCTC 9343 cultivated in defined medium (DM). Inset SEM image of the SERS substrate. The top image is a schematic of the detection principle and configuration for headspace SERS measurements. Photograph shows the experimental system used for in-situ monitoring of the culture headspace at $37^{\circ} \mathrm{C}$.

This band was assigned to the $v(\mathrm{C}-\mathrm{S})$ vibration of surface-bound methyl sulphide, $\mathrm{Ag}-\mathrm{S}-\mathrm{CH}_{3}$, on the basis of literature data. ${ }^{[19]}$ This was confirmed by GC-MS profiling of the headspace of the cultures (Supporting Information, Figure S3) which showed that dimethyl disulphide (DMDS) was present. DMDS spontaneously dissociates into methyl sulphide on $\mathrm{Ag}$ and $\mathrm{Au}$ surfaces ${ }^{[19]}$ and although it is present at much lower concentrations than other metabolites in the headspace of the bacteria, it presumably can be detected by SERS due to the preferential binding of the disulphide, which has a very strong affinity for $\mathrm{Au}$ and $\mathrm{Ag}$ surfaces. Subsequent studies over a larger $\mathrm{cm}^{-1}$ range also detected the $v(\mathrm{CH})_{\text {sym }}{ }^{[20]}$ vibration of the surface bound methyl sulphide, at $2923 \mathrm{~cm}^{-1}$, which is the only other strong band for methyl sulphide (Supporting Information, Figure S4). Another strong band was observed at $2148 \mathrm{~cm}^{-1}$ in the headspace SERS spectrum of $P$. aeruginosa. This could be assigned on the basis of literature data to Ag-C-N created from surface binding of hydrogen cyanide $(\mathrm{HCN})$, which is a well-known volatile biomarker for $P$. aeruginosa infections. ${ }^{[21],[22],[23]}$

SERS spectra of the headspace of sterile culture media spiked with DMDS at different concentrations were used to confirm that solution phase DMDS would give methyl sulphide SERS bands and allow the concentration level required to give the signals observed above the culture samples to be estimated. As shown in Figure 2, with the Ag substrate, the rate of signal growth varied with the DMDS concentration in the growth media, rising over a period of hours to a plateau value at $10^{-2} \mathrm{M}$ but rising much more slowly at $10^{-3} \mathrm{M}$, reaching less than $20 \%$ of the plateau value after several hours. These data can be fitted to a simple collision/adsorption model (Supporting Information) where the rate of increase of the signal is proportional to the DMDS spiking concentration (and thus the concentration in the headspace) and the fraction of the surface not already covered. In this model, the methyl sulphide is assumed to irreversibly bound and therefore the enhancing surface integrates the signal, so that it increases over time to a plateau value corresponding to full surface coverage. The only variables in the simulation are the plateau SERS intensity and an effective rate constant, which is a composite term combining the collision rate of DMDS with the surface and the proportion of these collisions which lead to adsorption as methyl sulphide. With $\mathrm{Ag}$, the plateau SERS intensity is high but the rate of dissociation of the disulphide on the surface is low, so that at a concentration of $10^{-3} \mathrm{M}$, the time taken for a significant amount of DMDS to react is hours. Conversely, as shown in Figure 2(d), Au substrates give lower absolute SERS signals but much faster kinetics, so that the time required to achieve a detectable signal is significantly shorter. These effects are well captured by the model which gives the simulated kinetic curves that are compared to the experimental data for both $\mathrm{Ag}$ and $\mathrm{Au}$ in Figure 2(c) and (d). The parameters from the simulation show a $10^{4} \mathrm{x}$ increase in rate but a $54 \mathrm{x}$ decrease in absolute signal (calculations shown in the Supporting Information) under the equivalent experimental conditions for Ag or Au substrates This means there is a choice between using $\mathrm{Ag}$ or Au depending on whether fast response or higher absolute signal is important.

Time dependent SERS of the headspace of a growing E. coli DH5a cultures showed the SERS signals of methyl sulphide increasing with time. This was due to a combination of the increase in the number of bacteria present (which was monitored through optical density, OD, of the culture) and the accumulation of methyl sulphide on the enhancing surface. The initial OD used was 0.03 , corresponding to $2.2 \times 10^{7} \mathrm{CFU} / \mathrm{ml}$. With the Ag substrate, the metabolite was first detected during the log phase of growth cycle and the SERS intensity followed the increasing $O D$ of the culture over 5 hours (Figure 3(c)). These measurements are different from the simple test systems shown above because the rate of signal increase is a convolution of the collision/adsorption rate and the concentration of bacteria. In duplicate runs, the rate of growth of the bacteria was different but this was also reflected in the time-dependent SERS spectra (see Figure S5). Inclusion of the OD 
measurements into the kinetic model is straightforward, since the fixed concentration of spiking DMDS can be replaced by the OD multiplied by a proportionality constant which reflects how the change of bacterial concentration alters the DMDS concentration in the growth medium. The results of this simulation are shown in Figure 3(c).

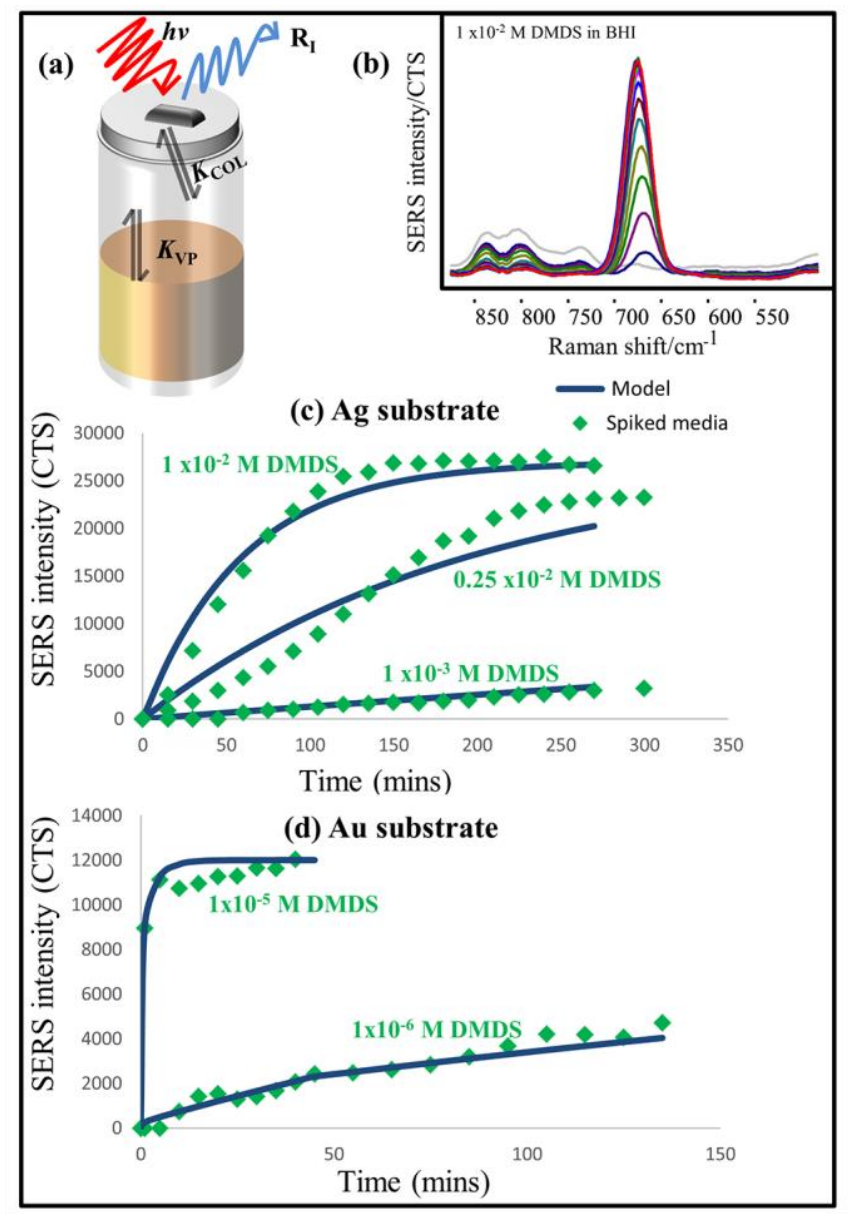

Figure 2[a]: (a) Illustration of the relationship between the equilibration of DMDS into the headspace and the adsorption of methyl sulphide onto the enhancing substrate. (b) Headspace SERS spectra of the time-dependent adsorption from BHI broth spiked with $1 \times 10^{-2} \mathrm{M}$ DMDS. Spectra recorded at 15 minute intervals with $\mathrm{Ag}$ substrate (c), (d) Growth of methyl sulphide SERS signal with time for $\mathrm{BHI}$ broth spiked with different concentrations of DMDS using (c) Ag substrate and (d) Au substrate. Solid lines are fits to data from the kinetic model discussed in the text. Accumulation times were $1 \mathrm{~s}$ and $20 \mathrm{~s}$ for $\mathrm{Ag}$ and $\mathrm{Au}$ substrates, respectively. Uncertainty (S.D) in SERS intensity was $4.8 \%$ for $\mathrm{Ag}$ and $9 \%$ for $\mathrm{Au}$.

The fact that this simple model which is fitted by rational parameters gives a reasonable fit to the experimental data suggests that it provides a good physical description of the sensing mechanism for the adsorption of DMDS. Calculation of the proportionality constant allows the DMDS concentration to be estimated at any bacterial concentration, so that at $\mathrm{OD}=0.5$ the [DMDS] in the broth was $1.4 \times 10^{-3} \mathrm{~mol} \mathrm{dm}^{-3}$.

When the sensing substrate was changed to $\mathrm{Au}$, the response was dramatically different, with the signal reaching half the plateau value just 1 hour after the start of incubation and rising to a plateau very early into the log phase. This was a consequence of the response of the Au substrate being considerably faster than the bacterial growth. The simulated data for the Au substrate given by the same model as was used for the Ag substrate is shown in Figure 3(d). In this case, the $50 \%$ plateau values corresponded to detection of bacteria which grew to a concentration of $5 \times 10^{7} \mathrm{CFU} / \mathrm{ml} 1$ hour after inoculation. At the noise levels associated with our instrumentation and a 20s exposure time, the minimum time for detection is $<15$ minutes, which corresponds to $<1.5 \times 10^{7} \mathrm{CFU} / \mathrm{ml}$. This detection time is unprecedented for a simple technique using a hand-held instrument. Moreover, it is important to stress that this is a nonoptimised figure. The detection limit depends on a complex interplay of the initial bacterial concentration, the rate of bacterial growth, the time which the detector is exposed to the headspace (and thus accumulates the chemisorbed product) and the Raman acquisition time, so the parameters could be adjusted to give lower detection limits or shorter detection times as required.
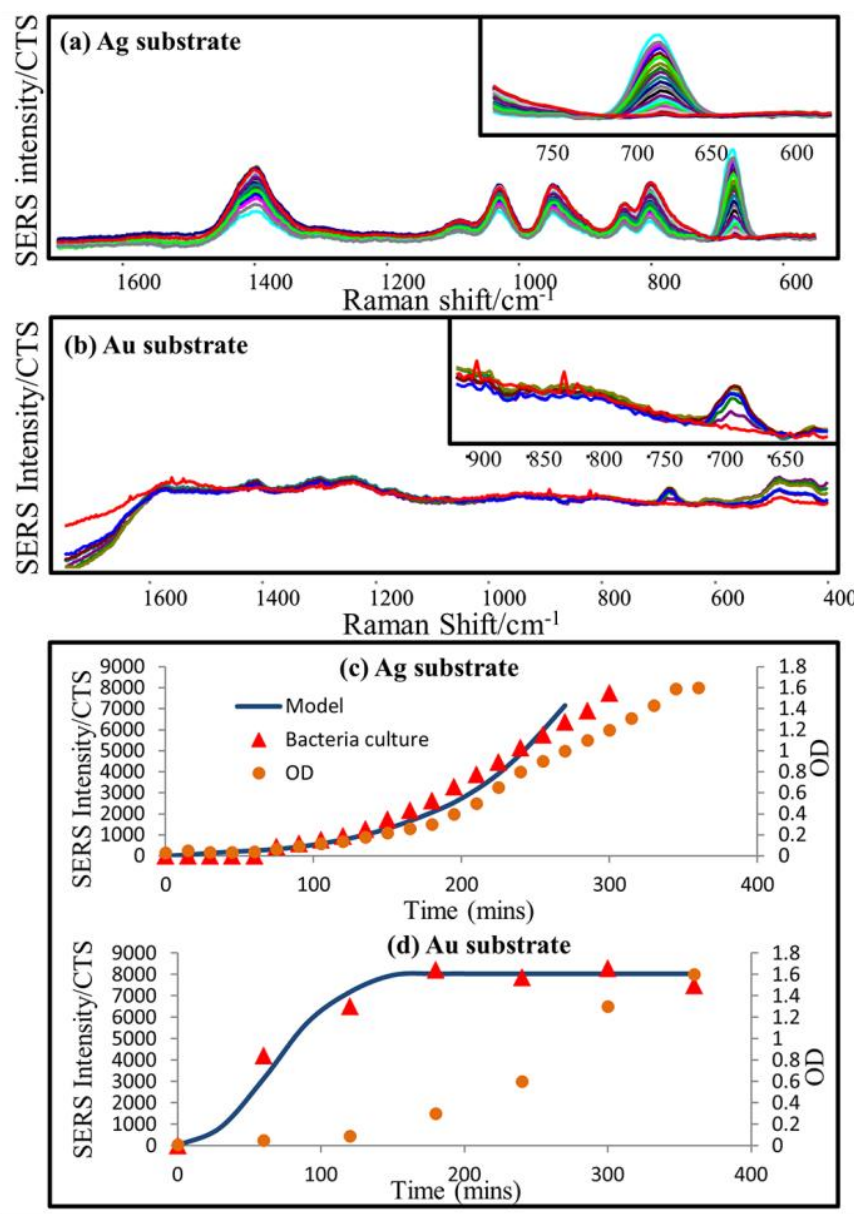

Figure 3: SERS spectra of kinetic run of the adsorption of methyl sulphide from E. coli culture on (a) Ag substrate (b) Au substrate. The inset spectra on the top right are expanded in the region of the methyl sulphide peak. (c) Comparison of the kinetic data showing bacterial growth (OD) and increasing methyl sulphide SERS intensity with time. Solid lines are fits to the SERS data using the kinetic model discussed in the text. (c) and (d) show comparable data for $\mathrm{Ag}$ and $\mathrm{Au}$ substrates, respectively. Uncertainty (S.D) in SERS intensity was $4.8 \%$ for $\mathrm{Ag}$ and $9 \%$ for $\mathrm{Au}$. 
Since the method detects metabolic activity, it can be used to determine if antibiotic treatment has been effective. For example, Figure 4 compares the headspace SERS spectra of a $10 \mathrm{ml}$ culture of $E$. coli DH5a which was grown to OD 0.13 (early log phase) and then split in two. One sample was treated with gentamicin, a commonly used broad spectrum antibiotic, while the other was a control. SERS spectra were then recorded for both samples at 1 hour intervals. In Figure 4(a), which shows the headspace SERS of the control sample, the intensity of the $v(\mathrm{C}-\mathrm{S})$ due to DMDS increased dramatically with time. A small amount of DMDS was also detected in the headspace of the antibiotic treated $E$. coli culture after 1 hour, presumably because DMDS continued to be produced up to the point where all the E. coli were killed. However, the SERS intensity of the DMDS peak did not increase in the same way as the control sample. In the control sample, the DMDS band grew very large

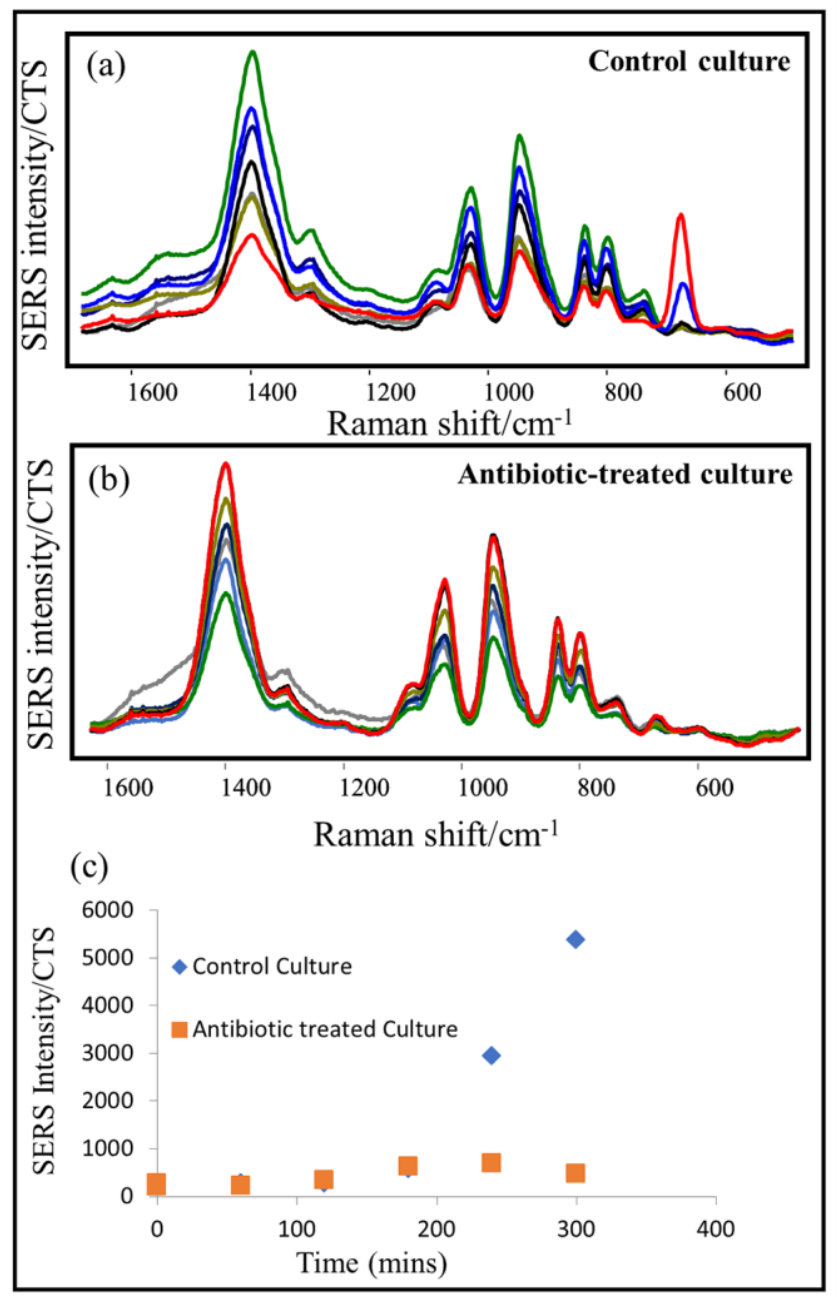

Figure 4: SERS spectra recorded at 1 hour intervals of the headspace of (a) $E$. coli DH5a (b) E. coli DH5a treated with $50 \mu \mathrm{g} / \mathrm{ml}$ gentamicin sulfate. The spectra have been averaged from 4 points across the Ag substrate.

at 5 hours but even at 4 hours there was already a detectable difference between the SERS intensity of the DMDS band in the antibiotic-treated and control samples. This is significantly faster than is possible with traditional culture-based methods. The results of a similar experiment in which the model culture was treated with an antibiotic (kanamycin) when it had grown to OD 0.34 are reported in the Supplementary Information (Figure S6). These results also show large differences in the SERS intensity between the antibiotic-treated and control samples, demonstrating that this method of determining the efficacy of antibiotic treatment is not confined to a single antibiotic or cultures in early log phase growth. This generality is important if the technique is to be used in clinical settings.

This work demonstrates that SERS can be successfully used to detect live bacteria through the headspace metabolites. This approach of using headspace SERS with hand-held instruments utilises all the major strengths of the technique: low cost, portability, selectivity for strongly adsorbed species and molecularly specificity. The fact that DMDS could be observed in the headspace of all 6 micro-organisms tested and 3 different growth media suggest that DMDS detection by SERS may be a general method for detection of metabolically active bacteria. This, combined with the speed of the measurements, raises the possibility that it may offer a new approach to rapid bedside detection of bacterial infections and rapid, high-throughput screening of antibiotics against specific infections.

\section{Experimental Section}

See Supporting Information.

\section{Acknowledgements}

This work was supported by funding from the Department of Education and Learning (DEL) and the EPSRC.

\section{Conflict of Interest}

The authors declare no conflict of interest

Keywords: Antibiotics $•$ Bacteria $・$ Headspace $・$ Nanotechnology SERS •

[1] J. O'Neill, Review on Antimicrobial Resistance: 'Rapid Diagnostics: Stopping unnecessary use of antibiotics': Wellcome Trust, 2015.

[2] H. M. Davey, Appl. Environ. Microbiol. 2011, 77, 5571-5576.

[3] G. A. Cangelosi, J. S. Meschke, Appl. Environ. Microbiol. 2014, 80, 58845891.

[4] P. Stiefel, S. Schmidt-Emrich, K. M-Weber, Q. Ren, BMC Microbiol. 2015 , 15,36 .

[5] M. Li, W. E. Huang, C. M. Gibson, P. W. Fowler, A. Jousset, Anal. Chem. 2013, 85, 1642-1649.

[6] J. Xu, D. Zhu, A. D, Ibrahim, C. C. R. Allen, C. M. Gibson, P. W. Fowler, W. E. Huang, Anal. Chem. 2017, 89, 13305-13312.

[7] Y. Tao, Y. Wang, S. Huang, P. Zhu, W. E. Huang, J. Ling, J. Xu, Anal. Chem. 2017, 89, 4108-4115. 
[8] B. J. Umber, H-W. Shin, S. Meinardi, S-Y. Leu, F. Zaldivar, D. M Cooper, D. R. Blake, Clin.Transl. Med. 2013, 2, 13.

[9] N. Kunze, J. Gopel, M. Kuhns, M. Jünger, M, Quintel, T. Perl, Appl. Microbiol. Biotechnol. 2013, 97, 3665-3676.

[10] J. Taucher, A. Hansel, A. Jordan, R. Fall, J. H. Futrell, W. Lindinger, Commun. Mass. Spectrom. 1997, 11, 1230-1234.

[11] J. N. Labows, K. J. McGinley, G. F. Webster, J. J. Leyden, J. Clin. Microbiol. 1980, 12, 521-526.

[12] W. Filipiak, A. Sponring, M. M. Baur, A. Filipiak, C. Ager, H. Wiesenhofer, M. Nagl, J. Troppmair, A. Amann, BMC Microbio. 2012, 12, 113.

[13] C. Zscheppank, H. L. Wiegand, C. Lenzen, J. Wimgender, U. Telgheder, Anal. Bioanal. Chem. 2014, 406, 6617-6628.

[14] R. Dutta, E. L. Hines, J. W. Gardner, P. Boilot, Biomed. Eng. Online. 2002, 1, 1-7.

[15] A. Pavlou, A. P. F. Turner, N. Magan, Lett. Appl. Microbiol. 2002, 35, 366369.

[16] J. W. Gardner, H. W. Shin, E. L. Hines, Sens. Actuator. B. Chem. 2000, 70, $19-24$.

[17] N. Itoh, S. E. J. Bell, Analyst. 2017, 142, 994-998.

[18] Y. Xu, M. P. Konrad, W. W. Y. Lee, Z. Ye, S. E. J. Bell, Nano Lett. 2016, 16, 5255-5260.

[19] L. L. Rouhana, M. D. Moussallem, J. B. Schlenoff, J. Am. Chem. Soc. 2011, 133, 16080-16091.

[20] J. K. Lim, I-H. Kim, K-H. Kim, K. S. Shin, W. Kang, J. Choo, S-W. Joo, Chem. Phy. 2006, 330, 245-252.

[21] D. Smith, P. Spanel, F. J. Gilchrist, W. Lenney, J. Breath. Res. 2013, 7,113.

[22] B. Enderby, D. Smith, W. Carroll, W. Lenney, Pediatr. Pulmonol. 2009, 2, 142-147.

[23] R. K. Lauridsen, L. M. Sommer, H. K. Johansen, T. Rindzevicius, S. Molin, L. Jelsbak, S. B. Engelsen, A. Bosie. Sci. Rep. 2017, 7, 45264.

[24] A. Kudelski, J. Raman. Spectrosc. 2003, 34, 853-862. 


\section{COMMUNICATION}

Detecting Bacteria: Headspace SERS allows selective detection of dimethyl disulphide above bacterial cultures. This volatile metabolic product can act as a marker for live bacterial cultures.

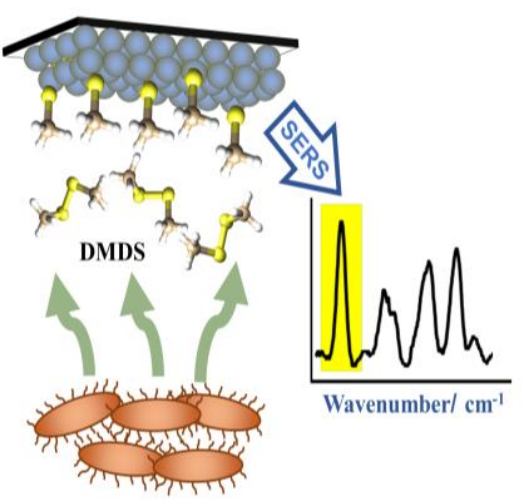

Jessica Kelly, ${ }^{\text {[a] }}$ Dr. Robin Patrick, ${ }^{\text {[a] }}$ Prof. Sheila Patrick, ${ }^{[\mathrm{b}]}$ Prof. Steven E.J. $B e / l[\mathrm{a}]^{*}$

Page No. - Page No.

Surface-enhanced Raman Spectroscopy for the Detection of Metabolic Products in the Headspace Above Live Bacterial Cultures 